\title{
A fast SWIR imager for observations of transient features in $\mathrm{OH}$ airglow
}

\author{
Patrick Hannawald ${ }^{1}$, Carsten Schmidt ${ }^{2}$, Sabine Wüst ${ }^{2}$, and Michael Bittner ${ }^{1,2}$ \\ ${ }^{1}$ Institute of Physics - University of Augsburg, Augsburg, Germany \\ ${ }^{2}$ German Remote Sensing Data Center - German Aerospace Center, Oberpfaffenhofen, Germany \\ Correspondence to: Patrick Hannawald (patrick.hannawald@ physik.uni-augsburg.de)
}

Received: 7 December 2015 - Published in Atmos. Meas. Tech. Discuss.: 14 January 2016

Revised: 11 March 2016 - Accepted: 16 March 2016 - Published: 4 April 2016

\begin{abstract}
Since December 2013 the new imaging system FAIM (Fast Airglow IMager) for the study of smaller-scale features (both in space and time) is in routine operation at the NDMC (Network for the Detection of Mesospheric Change) station at DLR (German Aerospace Center) in Oberpfaffenhofen $\left(48.1^{\circ} \mathrm{N}, 11.3^{\circ} \mathrm{E}\right)$.

Covering the brightest $\mathrm{OH}$ vibrational bands between 1 and $1.7 \mu \mathrm{m}$, this imaging system can acquire two frames per second. The field of view is approximately $55 \mathrm{~km}$ times $60 \mathrm{~km}$ at the mesopause heights. A mean spatial resolution of $200 \mathrm{~m}$ at a zenith angle of $45^{\circ}$ and up to $120 \mathrm{~m}$ for zenith conditions are achieved. The observations show a large variety of atmospheric waves.

This paper introduces the instrument and compares the FAIM data with spectrally resolved GRIPS (GRound-based Infrared P-branch Spectrometer) data. In addition, a case study of a breaking gravity wave event, which we assume to be associated with Kelvin-Helmholtz instabilities, is discussed.
\end{abstract}

\section{Introduction}

The $\mathrm{OH}$ airglow layer is located at a height of about $87 \mathrm{~km}$ with a half-width of approximately $4 \mathrm{~km}$ (e.g. Baker and Stair, 1988). It results from different chemical reactions leading to the emission of many vibrational-rotational lines in the visual and infrared optical range (for further details see e.g. Meinel, 1950; Roach and Gordon, 1973 and Rousselot et al., 2000). Observing the infrared emissions of the vibrationalrotational excited $\mathrm{OH}$ molecules offers a unique possibility for studying atmospheric dynamics. Atmospheric grav- ity waves are especially prominent features in the measurements.

Due to density decreasing exponentially with altitude, the wave amplitude of upward propagating waves increases as long as the wave is not dissipating energy. Therefore, small wave amplitudes in the tropopause can reach high amplitudes in the mesosphere and lower thermosphere region (MLT). It is widely accepted that propagating atmospheric gravity waves are important for the understanding of atmospheric dynamics and the energy budget of the atmosphere, as they provide the majority of the momentum forcing that drives the circulation in the MLT (Smith, 2012). A general overview of gravity waves and their importance for middle atmospheric dynamics can be found, besides others, in Fritts and Alexander (2003).

In order to acquire knowledge about gravity waves in this region, only a limited number of instruments can be used. Radars and especially lidars, for example, measure changes in temperature and wind with a comparatively high vertical resolution (see e.g. Sato, 1994; Chu et al., 2005; Dunker et al., 2015).

Besides these active instruments, passive spectrometers and imagers are frequently used to observe airglow emissions, which originate in the MLT region and are modulated by gravity waves. The Network for the Detection of Mesospheric Change (NDMC), for example, currently lists 57 observing sites, some of which are equipped with more than just one of these instruments. Spectrometers used for airglow observations are either space-borne (e.g. ENVISATSCIAMACHY; Bovensmann et al., 1999; von Savigny, 2015 and references therein) or ground-based (e.g. Nakamura et al., 1999; Schmidt et al., 2013; Noll et al., 2014). Airglow 
spectra are usually used for determining the atmospheric temperature at the height of the emission layer.

The spectral range of imaging instruments covers a wide range of airglow emissions - mostly at bandpasses between 500 and $1700 \mathrm{~nm}$, depending on the sensor material and applied filters. Many of these systems provide a large field of view (FOV) using fisheye lenses (see e.g. Taylor et al., 1995; Shiokawa et al., 1999; Smith et al., 2009 and Mukherjee et al., 2010). Others use a smaller aperture to focus on a distinct part of the night sky (e.g. Hecht et al., 2002 and Moreels et al., 2008). The temporal resolution typically varies from one image every few seconds to one every few minutes (see e.g. Hecht et al., 2002 or Taguchi et al., 2004). Another type of instrument related to both spectrometers and imagers are the MTM (Mesospheric Temperature Mapper; Taylor et al., 1999) and the AMTM (Advanced Mesospheric Temperature Mapper; Pautet et al., 2014), both of which use very narrowband filters to isolate individual emission lines, allowing the determination of airglow temperature during later processing.

The imager presented here provides a high temporal and spatial resolution in order to focus on small-scale and transient phenomena. In Sect. 2, the instrument and its set-up are described, and observations are compared to the infrared spectrometer system GRIPS 13, measuring in parallel direction to the imager at the same site. The data analysis is explained in Sect. 3, while the results of a case study are presented and discussed in Sect. 4.

\section{Instrumentation and operation}

In contrast to the design of most existing airglow imagers, our primary goal is to acquire images of the airglow layer at the fastest rate possible. Therefore, the system is designed to observe some of the brightest hydroxyl emission bands in the short wave infrared between approximately 1400 and $1650 \mathrm{~nm}$, utilising a sensitive detector as well as optics with high transmission.

The 320 pixel by 256 pixel sensor array of the FAIM 1Instrument (Fast Airglow IMager 1), based on InGaAs technology, has a spectral responsivity in the range from 950 to $1650 \mathrm{~nm}$ (model Xeva, manufactured by Xenics nv). It is equipped with a three-stage thermoelectric cooler and usually cooled to $235 \mathrm{~K}$ to reduce the dark current. Similar detectors are used by Pautet et al. (2014). The standard optics consist of an F\# 1.4 Schneider-Kreuznach SWIRON lens with a focal length of $23 \mathrm{~mm}$. In front of the lens is a mechanical shutter for protecting the sensor from aging processes due to direct sunlight during the daytime (see sketch in Fig. 1 for the instrument set-up).

In this set-up, standard exposure times of only $500 \mathrm{~ms}$ are used. The images are stored continuously, with a delay between two consecutive images due to readout and processing of about $10 \mathrm{~ms}$. This high temporal resolution enables the study of transient features in the airglow, providing the pos-

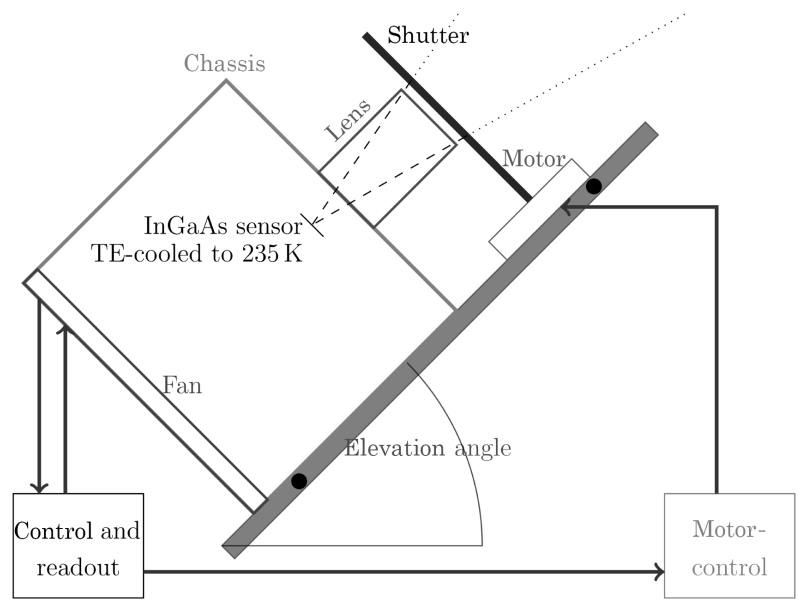

Figure 1. Sketch of the instrument and measurement set-up. See text for further details.

sibility to observe phenomena with frequencies significantly higher than the Brunt-Väisälä frequency, e.g. infrasound (see e.g. Pilger et al., 2013) or turbulence.

The airglow signal is converted into 12-bit greyscale images with values ranging from 0 to 4095 , hereafter denoted as counts or arbitrary units (a.u.). A series of 100 darkened images with the same exposure time is acquired in order to determine the noise with the current settings. The standard deviation of the mean value of each image results in 25 counts.

Before analysis, all images are flat-fielded by being measured in front of a homogeneous large-area black-body source. This procedure eliminates the so-called fixed pattern noise as well as the vignetting of the lens.

The set-up of FAIM 1 is shown in Fig. 2. The instrument is operated at a zenith angle of $45^{\circ}$. The lens provides a field of view (FOV) of $20^{\circ} \times 24^{\circ}$ with a barrel distortion of less than $1 \%$, which is neglected. Due to the fact that the instrument is not looking into zenith direction, the resulting area observed is trapezium-shaped, with a size of about $55 \mathrm{~km} \times 60 \mathrm{~km}$ at the altitude of the $\mathrm{OH}$ emission peak, at $87 \pm 4 \mathrm{~km}$ (height according to Baker and Stair, 1988). The instrument is located at Oberpfaffenhofen, Germany $\left(48.09^{\circ} \mathrm{N}, 11.28^{\circ} \mathrm{E}\right)$ with an azimuth angle of $214 \pm 1^{\circ}$ (direction SSW). The observed area is located above parts of southern Germany and the Austrian Alpine region (see Fig. 4c).

The observed trapezium-shaped area of the airglow layer is the projection of the rectangular-shaped sensor due to the observation geometry. As a result of this, the images show a distorted view of the airglow layer and have to be corrected to get an equidistant scale, which is necessary for the following analyses. Therefore, a transformation is applied to the images to remap the pixels to the original shape. This transformation, based on some trigonometrical considerations, is mainly dependent on the zenith angle and the FOV. The left-hand side of Eq. (1) gives the geographical (Cartesian) coordinates de- 


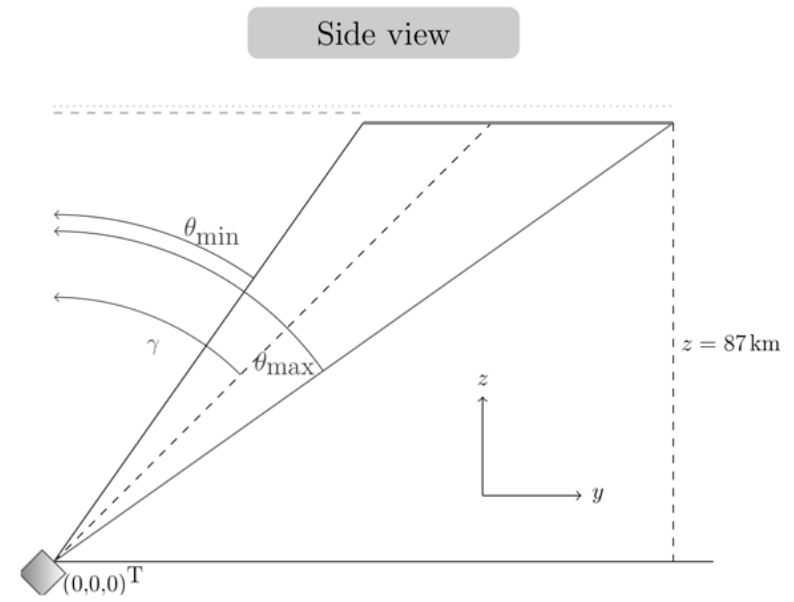

\section{Aerial perspective}

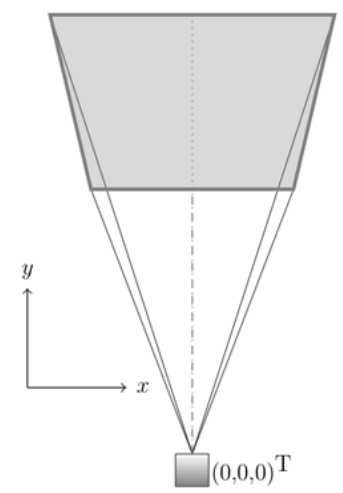

Figure 2. Measurement set-up with a zenith angle of $45^{\circ}$ viewed from the side and from an aerial perspective. The axes show the definition of the coordinate system used to reference the pixels to geographical coordinates. The instrument is located at the centre of the coordinate system, $z=87 \mathrm{~km}$ is the altitude of the $\mathrm{OH}$ airglow layer, $\gamma$ is the zenith angle of the instrument and $\theta$ is the variable in the range of $\gamma \pm \mathrm{FOV}_{\text {vertical }}$, FOV being the aperture angle of the instrument of $10^{\circ}$ (vertical) and $12^{\circ}$ (horizontal).

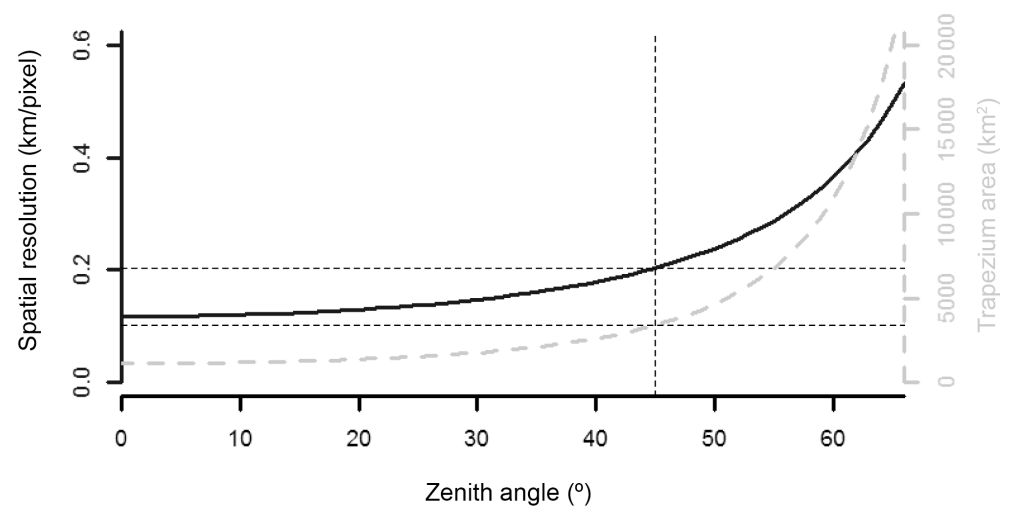

Figure 3. Mean spatial resolution of the acquired images as a function of zenith angle (black). The grey dashed line shows the respective area of the trapezium-shaped FOV with the axis on the right side of the graph. The standard angle of $45^{\circ}$ is marked by thin dashed lines for both curves, showing a mean spatial resolution of $200 \mathrm{~m}$ and a corresponding area of $3400 \mathrm{~km}^{2}$.

pending on the zenith angle $\theta \in\left(45^{\circ} \pm \frac{19.5^{\circ}}{2}\right)$ and the azimuth angle $\phi \in\left( \pm \frac{24.1^{\circ}}{2}\right)$ for each pixel:

$$
\left(\begin{array}{c}
x(y(z, \theta), z, \phi) \\
y(z, \theta) \\
z
\end{array}\right)=\left(\begin{array}{c}
\sqrt{y^{2}+z^{2}} \cdot \tan (\phi) \\
z \cdot \tan (\theta) \\
87 \mathrm{~km}
\end{array}\right),
$$

with Cartesian coordinates $x, y$ and $z ; y$ is parallel to the line of sight and $x$ is perpendicular to it (see Fig. 2). The airglow layer is assumed to be at constant altitude $z=87 \mathrm{~km}$. The origin is given by the location of the instrument.

After the geographical coordinates for each pixel have been determined, the area covered by the entire image is calculated. It amounts to approximately $3400 \mathrm{~km}^{2}$ or $60 \mathrm{~km} \times 55 \mathrm{~km}$ (height and width of the trapezium). For the $320 \times 256$ pixel array used, this refers to an approximate resolution of $200 \mathrm{~m} \times 200 \mathrm{~m}( \pm 10 \%)$ pixel $^{-1}$. The relation be- tween the zenith angle and the mean spatial resolution is shown in Fig. 3 as a thick solid line. The grey dashed line shows the size of the observed area. The standard zenith angle of $45^{\circ}$ is marked by thin dashed lines. In zenith direction, the mean spatial resolution is $120 \mathrm{~m}$ with the current optics.

The uncertainty depends on several contributions. First of all the airglow layer height of $87 \mathrm{~km}$ is a statistical mean value and may vary significantly. According to Baker and Stair (1988) the variation is $\pm 4 \mathrm{~km}$. Furthermore, the accuracy of the measurement set-up is limited to $\pm 0.5^{\circ}$ concerning the zenith angle, and $\pm 0.3^{\circ}$ and $\pm 0.2^{\circ}$ concerning the aperture of the lens. This results in an overall uncertainty of $\pm 400 \mathrm{~km}^{2}$ or about $12 \%$ for the covered area and an uncertainty of $\pm 24 \mathrm{~m} \mathrm{pixel}^{-1}$ respectively. Since the uncertainty is dominated by the variability of the airglow layer height, these numbers are taken as a measure of precision, although the observational set-up only limits the accuracy of the mea- 


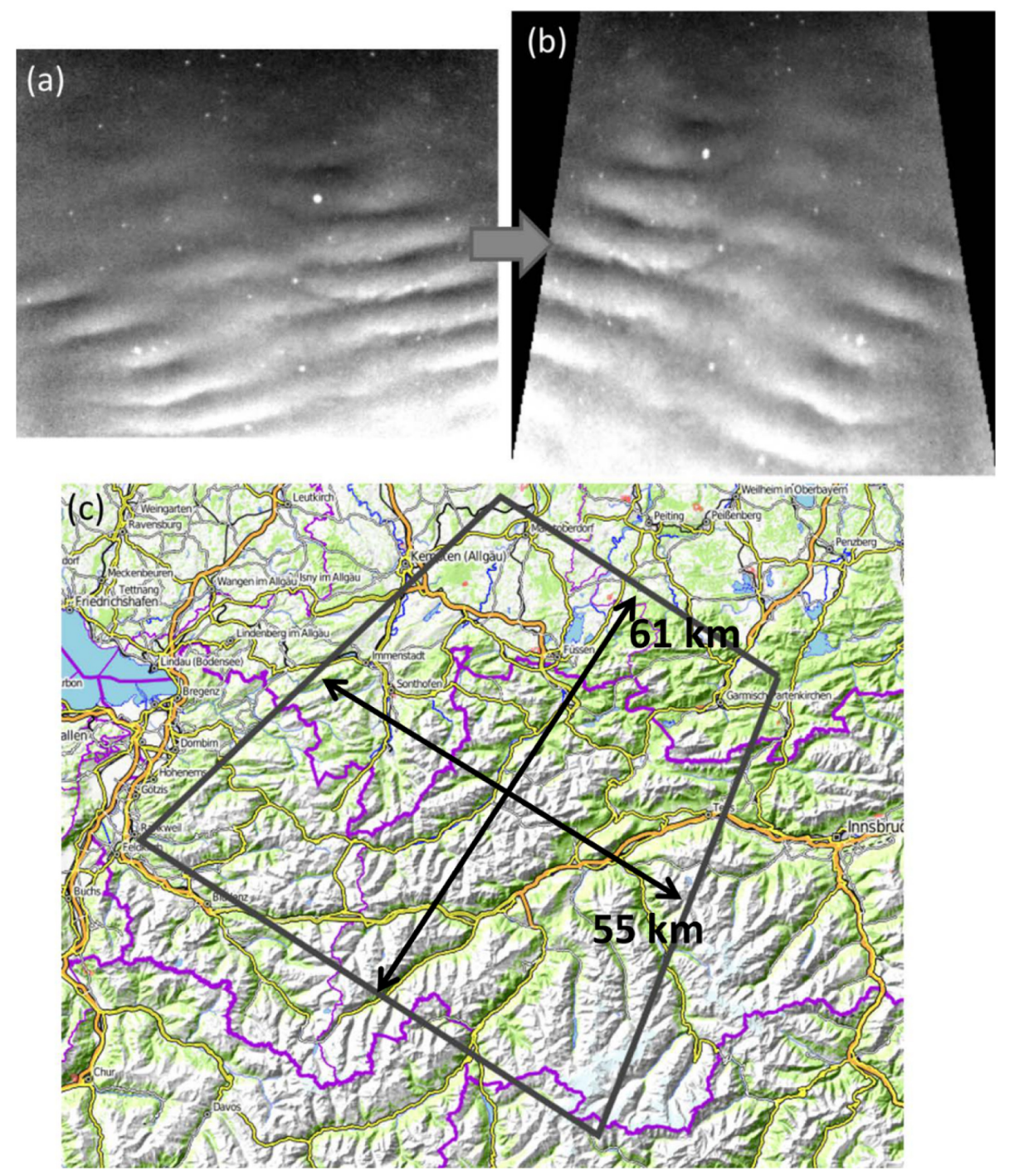

Figure 4. (a) shows the flat-field corrected image, which is distorted due to the geometry of the measurement set-up, with a zenith angle of $45^{\circ}$. In (b) this distortion is corrected by Eq. (1). Additionally, the image is mirrored onto the middle axis to change the ground-based observer's view to a satellite perspective. (c) shows the position of the FOV within the Alpine region (source: www.opentopomap.org, October 2015).

surements. If just considering the variability of the airglow layer the uncertainty for the visible area is $300 \mathrm{~km}^{2}$ and consequently $\pm 18 \mathrm{~m} \mathrm{pixel}^{-1}$.

However, for geographically mapping the pixels, each pixel is assigned a preliminary coordinate based on Eq. (1). A new equidistant grid is then constructed with a scale equal to the mean spatial resolution of $200 \mathrm{~m}$ and the preliminary coordinates are transformed to the new grid. This new grid is 320 by 306 pixels in the described set-up. This results in some empty rows in the corrected image near the horizon where the available values are further apart, and more values available for one new grid point near the zenith where the original values are closer together. In the former case, the missing values are interpolated by taking the mean value of up to eight non-empty nearest neighbours. In the latter case, the mean of all available original values within the new grid point is calculated. Additionally to this mapping, the image is mirrored onto the $y$ axis to change the view from a groundbased perspective to a satellite perspective. Figure 4 a shows the raw (flat-field corrected) image. After the transformation and remapping we obtain the geographically corrected image (b). According to this, a wave field can easily be referred to a map (compare Fig. 4c).

In 2014 the instrument FAIM 1 was operated for 350 nights with the described set-up. Measurements are taken for solar zenith angles larger than $96^{\circ}$. For each night, one row and one column of about 1000 images is taken and plotted versus time. These so-called keograms can easily be used to 


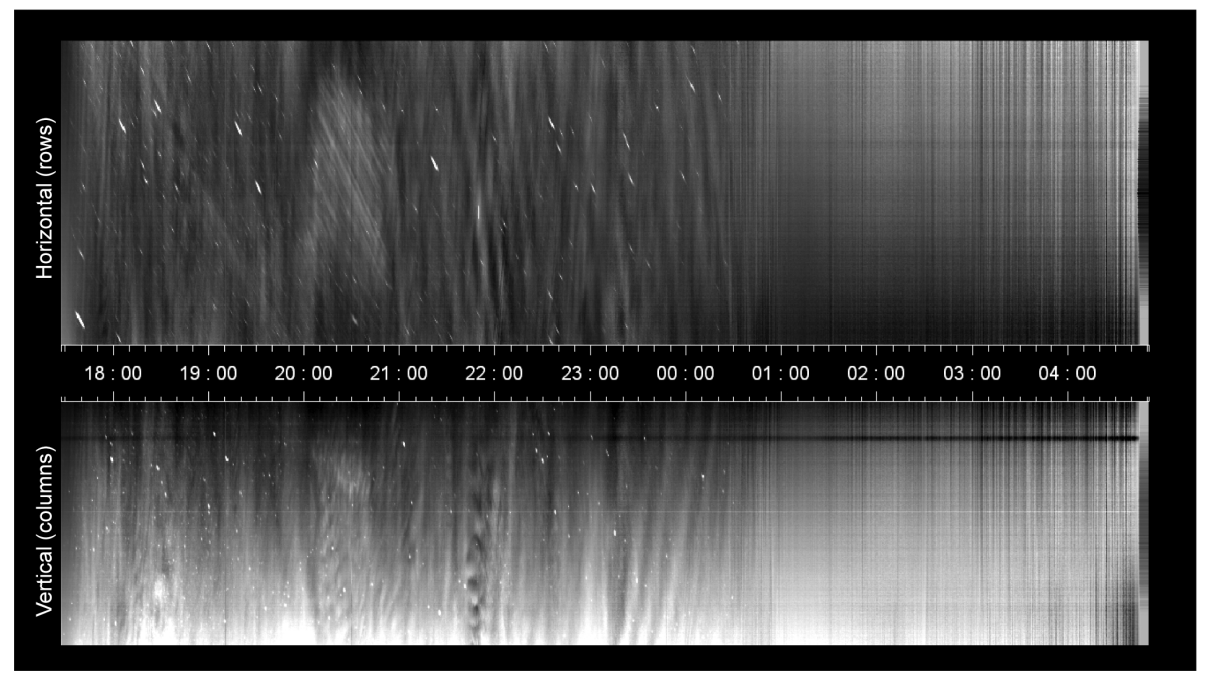

Figure 5. Keogram for one row and one column of images for the night of 3 to 4 October 2014. Between 17:30 and 00:40 UTC stars can be seen clearly, but after 00:40 UTC dense cloud cover appears. Several wave events can easily be identified at 18:30, 20:00-21:00, 22:00 and 23:30-00:30 UTC. The time interval between 21:05 and 22:30 UTC is further investigated in Sects. 3 and 4.

obtain information about cloudiness, incident moonlight or high atmospheric wave activity.

As a typical example, Fig. 5 shows keograms for the night from 3 to 4 October 2014. Between 17:30 and 00:40 UTC there is a fairly clear sky since stars are visible in the keograms, which is confirmed by the respective video sequences of this night. From 00:40 UTC onwards there are high-density clouds, which completely inhibit airglow observations.

Since FAIM 1 covers a rather broad spectral range from 950 to $1650 \mathrm{~nm}$, several intercomparisons with co-located GRIPS systems have been performed. These instruments usually acquire airglow spectra between 1.5 and $1.6 \mu \mathrm{m}$, but they can be adjusted to record any other part of the airglow spectrum between approximately 0.9 and $1.65 \mu \mathrm{m}$. Usually, $\mathrm{OH}(3-1)-\mathrm{P}-$ branch spectra acquired with these spectrometers are used to derive rotational temperatures with a temporal resolution of $5 \mathrm{~s}$ (GRIPS 13) or $15 \mathrm{~s}$ (GRIPS 16) (see Schmidt et al. (2013) for further details). For the investigation of the FAIM performance both spectrometers GRIPS 13 and GRIPS 16 were operated parallel to FAIM 1. The FOV of GRIPS $13\left(15^{\circ} \times 15^{\circ}\right)$ is comparable in terms of size to the FOV of FAIM 1, whereas the FOV of GRIPS 16 is significantly smaller $\left(2^{\circ} \times 2^{\circ}\right)$. In order to match the FOV of GRIPS 13, the two-dimensional greyscale images of FAIM 1 are reduced to their mean value over the slightly smaller FOV of the spectrometer. On the other hand, airglow spectra are integrated to yield one intensity value for each spectrum. Both time series are then averaged to get the same temporal resolution of $1 \mathrm{~min}$. Since neither instrument was absolutely calibrated at this time, both time series have been normalised independently to their individual maximum intensities.
Figure 6 shows the intensity time series, again for the night of October 3 to 42014 until midnight, when clouds started to appear. The upper panel refers to the night from the beginning of the measurement, whereas the lower panel does not consider the first $25 \mathrm{~min}$ of twilight data. The time series appear to be anti-correlated during this time. Therefore, the correlation coefficient increases from 0.15 to 0.87 when avoiding twilight. Investigations of other nights in the same manner (not shown) reveal even higher correlation coefficients up to 0.99 . The discrepancy at dusk conditions is due to the emission of $\mathrm{O}_{2}(0-0)$ at $1.27 \mu \mathrm{m}$, which decreases exponentially after sunset (see e.g. Mulligan and Galligan, 1995).

Since the $\mathrm{O}_{2}(0-0)$ emission originates from different (variable) heights compared to $\mathrm{OH}$ and exhibits a rather long half-value time of approximately $1 \mathrm{~h}$, the behaviour after sunset is further investigated in order to estimate its impact on the observations. Hence, parallel measurements with FAIM 1, GRIPS 13 and GRIPS 16 have been taken, with GRIPS 13 adjusted to observe the $1.27 \mu \mathrm{m}$ emission and GRIPS 16 limited to the integrated OH(3-1)-Q-branch intensities (around $1.51 \mu \mathrm{m}$ ) to also avoid the weaker $\mathrm{O}_{2}(0-1)$ emission at $1.58 \mu \mathrm{m}$.

Figure 7 shows the different evolution of these three intensities normalised to their individual maximums. The start of each time series is marked with dashed lines. The $\mathrm{O}_{2}(0-$ 0 ) intensity at $1.27 \mu \mathrm{m}$ (black) shows the expected exponential decay after sunset, which is investigated in substantial detail by Mulligan and Galligan (1995) and almost no other small-scale variation. The $\mathrm{OH}(3-1)$ intensity (blue) also shows the expected behaviour with rising intensities from 18:00 to 19:00 UTC, caused by increasing ozone concentrations, involved in the formation of excited $\mathrm{OH}$. However, short period variations clearly dominate after 18:45 UTC. 

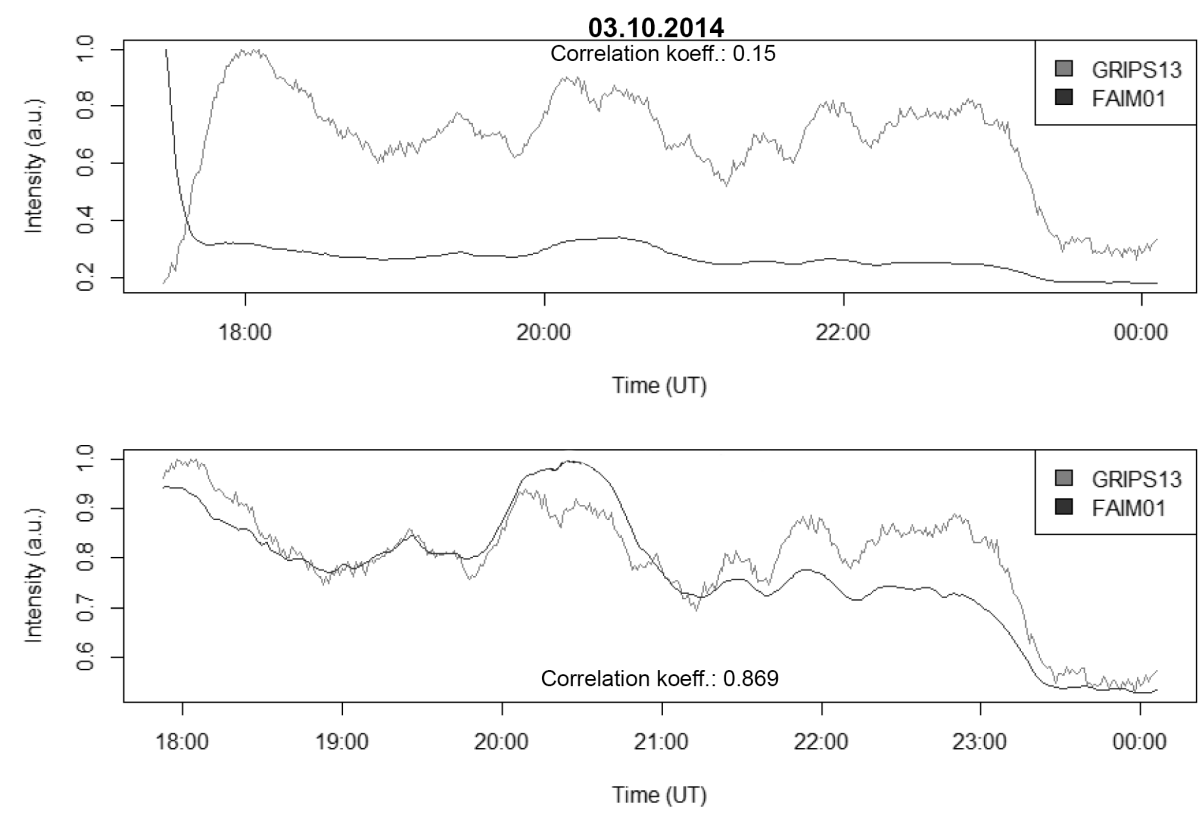

Figure 6. Intensity time series for the night of 3 to 4 October 2014. The black line shows the mean value over all pixels of FAIM 1 within the FOV of GRIPS 13 and the grey line shows the intensity measured with GRIPS 13 integrated between 1500 and $1600 \mathrm{~nm}$. Both data sets are averaged to $1 \mathrm{~min}$ mean values to avoid effects of different sampling rates and normalised to their individual maximums. Top: the entire time interval from the start of the measurement until 00:00 UTC when clouds emerged. A correlation of only 0.15 is determined. Bottom: same as above, but the first $25 \mathrm{~min}$ of twilight are avoided. The correlation now increases to 0.87 .

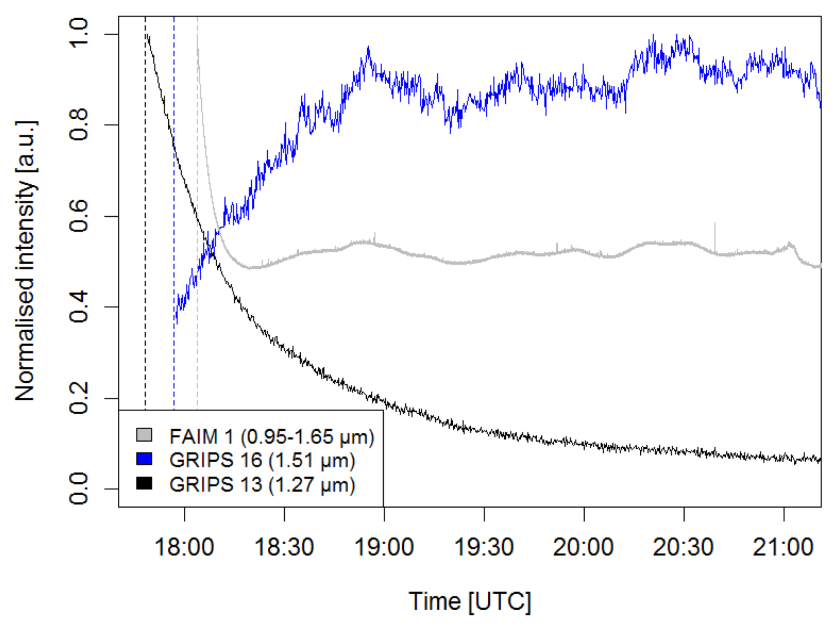

Figure 7. Measurements in the parallel direction of FAIM 1 with spectral range from 950 to $1650 \mathrm{~nm}$, GRIPS 13 adjusted for measuring $1.27 \mu \mathrm{m} \mathrm{O}_{2}(0-0)$-transition and GRIPS 16 for the $\mathrm{OH}(3-1)$ Q-branch-transition at $1.51 \mu \mathrm{m}$ for the first $3 \mathrm{~h}$ of the night. The case study shows an exponential decay of $\mathrm{O}_{2}$ and an increase of $\mathrm{OH}$ intensity. The FAIM time series (averaged over the FOV of GRIPS 13) shows a mixture of both behaviours.

The evolution of the intensities recorded by FAIM 1 includes both oxygen and hydroxyl emissions. However the influence of $\mathrm{OH}$ - including a wide range of emissions between 0.9 and $1.65 \mu \mathrm{m}$ - appears to be rather strong. After a sharp and short decrease in intensity directly after sunset, short periodic variations similar to the pure $\mathrm{OH}$ emissions recorded by GRIPS 16 dominate the temporal evolution after 18:20 UTC. Obviously, the influence of the $\mathrm{O}_{2}$ emission on the observation is of shorter duration than its half-value time of about $1 \mathrm{~h}$. This result has been validated by using a longpass filter with $1260 \mathrm{~nm}$ cut-on wavelength in the FAIM set-up (not shown). However, it does not improve the observations significantly; on the contrary it also excludes large portions of the $\mathrm{OH}$ emissions. Therefore, it is not used in the regular observational set-up any more.

\section{Data analysis}

Data from the keograms of the night from 3 to 4 October 2014 shown in Fig. 5 are chosen for a case study. The time period of roughly one and a half hours between 21:05 and 22:30 UTC is investigated. The entire data set used for the analysis consists of 10000 images in this time interval. To illustrate the wave structures, a series of images with a time difference of about $4 \mathrm{~min}$ between each of them is shown in Fig. 8 . The series reveals an emerging wave structure (wave I) appearing and disappearing within about $20 \mathrm{~min}(21: 40$ to 22:00 UTC), clearly recognisable in the images (7) to (11). The black dashed line marks the approximate direction of the wave vector. This line is used as a transverse section through the images for further analysis. 

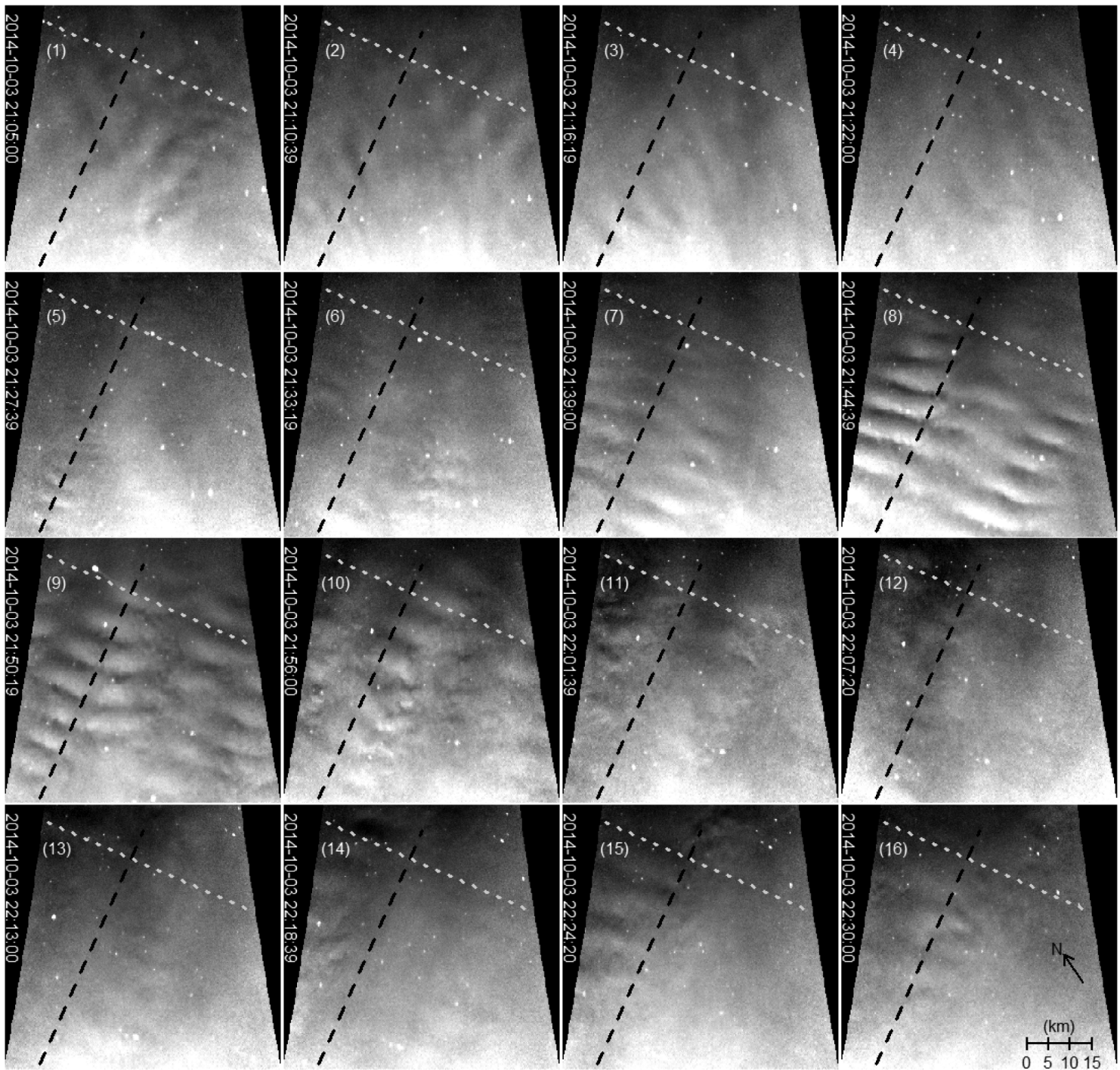

Figure 8. Series of consecutive images within the chosen time interval from 21:05 to 22:30 UTC. The black dashed line indicates the transverse section through the images used to analyse the smaller-scale wave (I). The grey dotted line shows a transverse section approximately in direction of the wave vector for the investigation of a faint larger wave structure (II) almost perpendicular to the first one. The whole observed area is about $55 \mathrm{~km}$ (central width of the FOV) $\times 60 \mathrm{~km}$ (height of the FOV).

A second wave (wave II) superimposes on wave (I) with a wave vector almost exactly perpendicular to wave (I). It is not as easily recognisable in the images as the first wave, but Fig. 9 shows it without a doubt. The grey dotted line marks the direction of the wave vector of this wave (II). The superposition can best be seen in the images (7) to (11). Images (9) to (11) show the presence of even smaller-scale structures of less than $2 \mathrm{~km}$. Similarly images (1) to (6) show a variety of different waves not further analysed here.

An intensity gradient is superimposed on each image, causing the upper part (low zenith angle) of the images to be darker than the lower (high zenith angle). This is due to the van Rhijn effect: the larger the zenith angle of the set-up, the longer the line of sight through the airglow layer. This results in systematically higher recorded intensity. It is corrected with the formula derived by van Rhijn (1921) based on geometrical considerations, used here in the representation given by Roach and Gordon (1973):

$$
\mathrm{I}(\theta)=\frac{1}{\sqrt{1-\left(\frac{R}{R+h}\right)^{2} \cdot \sin ^{2}(\theta)}},
$$

with zenith angle $\theta \in\left(45^{\circ} \pm 10^{\circ}\right)$, earth radius $R(6371 \mathrm{~km})$ and an airglow layer height $h$ of $87 \mathrm{~km}$. The correction factor for each row of the original rectangular image is the following:

$\mathrm{I}_{\text {row, new }}(\theta)=\mathrm{I}_{\text {row, old }} \cdot \frac{\mathrm{I}(\theta)}{\mathrm{I}(\gamma)}$, 

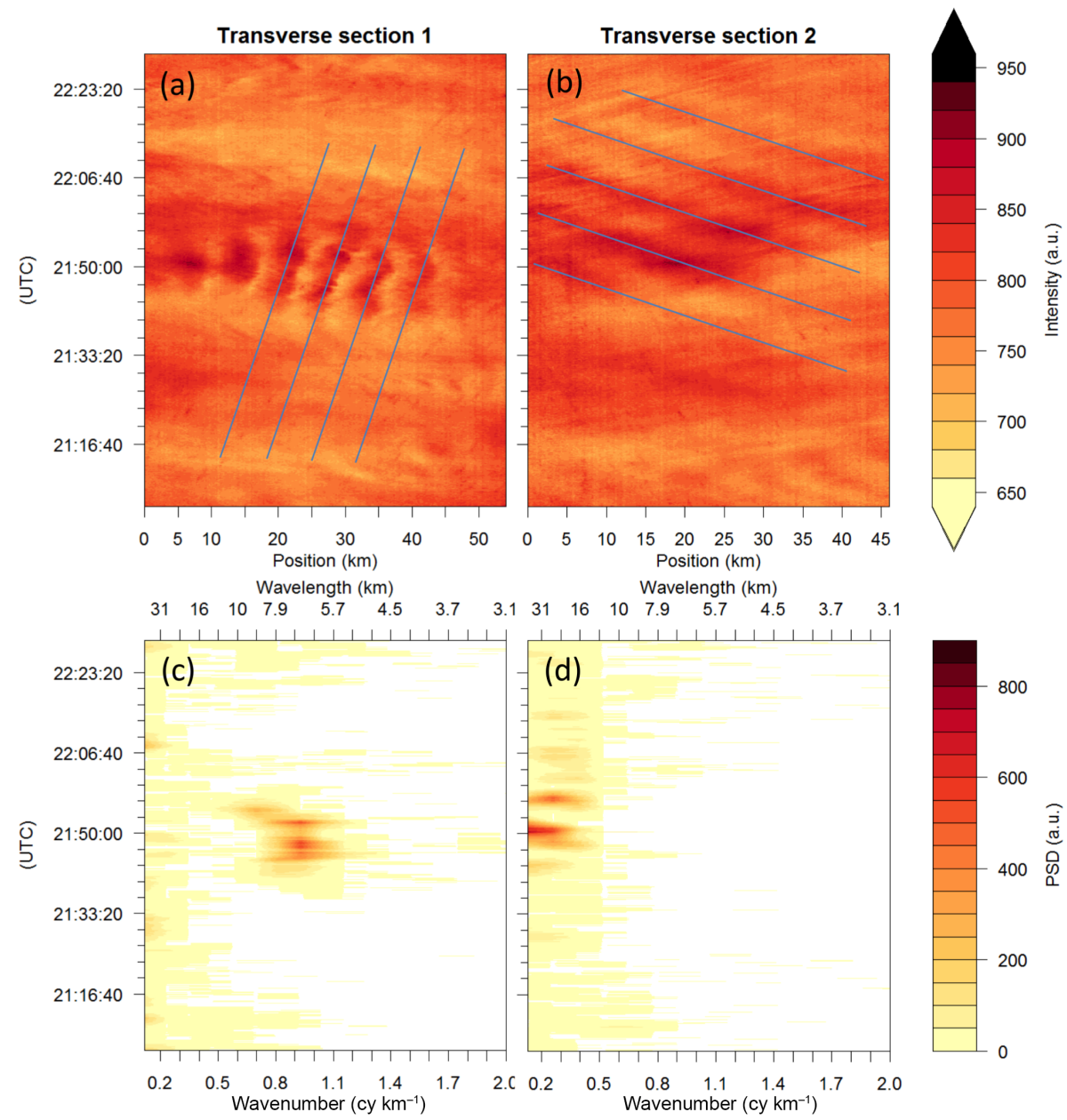

Figure 9. (a, b) show the temporal evolution of the transverse sections of wave (I) and wave (II). The lines indicate the position of the wavefronts. The abscissa corresponds to the position within the transverse sections shown in Fig. 8 (axis origin corresponds to the upper part of the transverse sections). Panels (c, d) show the Fourier transforms of each line of (a, b) respectively. The white areas are not significant on a $95 \%$-level.

where $\mathrm{I}_{\text {row, new }}$ is the corrected (new) intensity of the original signal ( $\mathrm{I}_{\text {row, old }}$ ) recorded by one row of pixels and $\gamma$ is the zenith angle of the instrument's optical axis of $45^{\circ}$ (see Fig. 2).

Further preprocessing includes removing the stars in the images to avoid a potential influence on later spectral analyses. They are identified by applying a gaussian blur on the image and subtracting this modified image from the original one. The gaussian blur is very sensitive for small structures with a strong intensity gradient - as is the case with stars. All pixels above a predefined threshold will be treated as star pixels and further investigated to identify the radius of each star. For each star, all pixels lying within this radius around the pixel with the highest intensity are identified as star pixels and removed from the original image. Finally, the missing values for these pixels are interpolated over nearest neighbours of non-star pixels. The method works very well for bright stars. Some stars with an intensity only slightly above the background (airglow) intensity, resulting in an intensity below the threshold level, remain in the images, but do not have a large impact on the result of subsequent spectral analyses.

After these corrections the above-mentioned wave events are analysed by extracting data along the transverse sections shown in Fig. 8, obtaining 10000 such sections for each wave. This is actually similar to taking a keogram for each propagation direction with a high temporal resolution.

Figure 9a and $\mathrm{b}$ depict the contour plots, with ordinates indicating the time in UTC and abscissas indicating the position along the transverse sections in kilometres (starting at the top part of the sections, compare Fig. 8). In order to determine the wavelengths from Fig. $9 \mathrm{a}$ and $\mathrm{b}$, some guiding lines are drawn along the apparent wavefronts. The distance between two of these lines in direction of the abscissa yields the wavelength and the ordinate distance yields the period of the wave structures. Uncertainties of $\pm 2 \mathrm{~km}$ for the wavelengths 
and of $\pm 100 \mathrm{~s}$ for the period are derived due to ambiguities of the positions of the guiding lines.

The wave parameters are also derived in Fourier space. Therefore, after removing linear trends and multiplication with a Hann window, the fast Fourier transformation (FFT) is applied for each time step shown in Fig. 9a and b.

The significance is derived by comparing 100 data sets with random numbers with the same mean and standard deviation as the original one, for each line. The insignificant values are indicated by white areas in the spectrograms Fig. 9c and d. It should be mentioned that, especially for larger wavelengths, the uncertainty retrieved from the FFT will be higher, because of the discrete sampling bins and the calculation of the wavelength from the wavenumbers.

\section{Results and discussion}

During the night from 3 to 4 October 2014, two prominent wave events could be identified (see Fig. 8). For the smaller wave (I) a horizontal wavelength of about $6.8 \mathrm{~km}$ is determined on the basis of the wavenumber in the Fourier domain during the time interval from 21:36 to 21:54 UTC (see Fig. 9c). Its respective uncertainty amounts to $\pm 0.4 \mathrm{~km}$. Afterwards, from 21:54 to 21:56 UTC, the wavelength appears to shift to about $9.0 \mathrm{~km}$ (uncertainty range: 8.4 to $9.9 \mathrm{~km}$ ) before it fades. If considering the transverse sections of wave (I) instead (Fig. 9a), the wavelength can be determined to $7.0 \pm 2.0 \mathrm{~km}$ (with support of the guiding lines); the change in wavelengths, however, appears to be insignificant.

The main horizontal wavelength of wave (II), determined in the frequency domain, is $24.0 \mathrm{~km}$ (uncertainty range: $20 \mathrm{~km}$ to $36 \mathrm{~km}$, see Fig. 9d). Since the resolution for small wavenumbers is rather imprecise in Fourier space, its wavelength can be determined more precisely in position space (Fig. 9b), yielding in $20.2 \pm 2.0 \mathrm{~km}$. Apparently, the uncertainty of the wavelength is smaller for wave (I) when analysed in the frequency domain, but it is the opposite for wave (II). The values are summarised in Table 1.

While wave (I) can be observed for about $20 \mathrm{~min}$, wave (II) is apparent in the Fourier spectrum (Fig. 9d) for about $35 \mathrm{~min}$ from 21:40 to 22:15 UTC. The Fourier amplitude maximises at 21:51 UTC, just when wave (I) is starting to diminish.

The period of wave (I) amounts to $1400 \pm 100 \mathrm{~s}$ determined by the ordinate distance of the guiding lines in Fig. 9a. Wave (II) has a period of $585 \pm 100 \mathrm{~s}$ (see Fig. 9b).

Thus, the phase velocity can be calculated according to the following equation:

$v_{\text {phase, horizontal }}=\frac{\lambda}{T} \pm\left(\frac{\Delta \lambda}{T}+\frac{\lambda}{T^{2}} \cdot \Delta T\right)$,

with the period $T \pm \Delta T$ and the horizontal wavelength $\lambda \pm \Delta \lambda . \Delta \lambda$ is either the uncertainty of $2.0 \mathrm{~km}$ in position space (referring to $\lambda_{1}$ ) or half the size of the specific uncertainty range (referring to $\lambda_{2}$ ). As discussed before, the analysis in frequency domain is more suitable for wave (I), whereas for wave (II) the analysis in position space exhibits lower uncertainty. Considering this, wave (I) propagates with a phase velocity of $4.9 \pm 0.6 \mathrm{~m} \mathrm{~s}^{-1}$ and wave (II) with $34.5 \pm 9.3 \mathrm{~m} \mathrm{~s}^{-1}$.

We speculate that the small wavelength, short lifetime and perpendicular direction of propagation may indicate a ripple structure as defined by Adams et al. (1988) and Taylor and Hapgood (1990). They are distinguished from larger-scale structures termed as bands. Ripples are strongly related to Kelvin-Helmholtz instabilities (KHIs) and convective instabilities (Hecht, 2004). Images (9) to (11) of Fig. 8 show further small-scale features, which we assume to be KHI billows. Fritts et al. (2014) show structures based on model calculations of the $\mathrm{OH}$ airglow response to KHIs looking very similar to these. Yamada et al. (2001) and Hecht et al. (2014) present similar phenomena in their measurements. The latter provide a detailed analysis combining the measurements of different instruments.

In order to investigate potential influences of these smallscale waves on mesopause temperatures, the upper panel of Fig. 10 shows the variation of rotational temperatures observed with GRIPS 13 at the same time, shown as 1 min running mean values and an original resolution of $5 \mathrm{~s}$. During this time span the instrument measured in parallel direction with FAIM 1. The mean temperature in the observed time range is $205.7 \mathrm{~K}$. The thick smooth line represents a spline of the time series revealing the underlying long-period structure (filtering periods lower than about $1000 \mathrm{~s}$ ) and is included to guide the eye. Obviously, two wave crests can be seen in the temperature, dissolving after 22:10 UTC.

The bottom panel of Fig. 10 shows the Fourier transform of the time series, and the dashed line gives the 0.95 confidence level calculated for each frequency, based on 10000 random number time series with same mean and standard deviation as the original time series. Significant oscillations are identified at periods of $2500,1000,510$ and $390 \mathrm{~s}$. The periods found in the FAIM images and the periods of the GRIPS temperatures are difficult to match. A reason for that is observational selection, meaning that, on the one hand, the small wave structure (I) in the images has several wave crests and troughs within the FOV of GRIPS, which cannot resolve it properly because its spatial resolution is similar to the size of the whole FOV of FAIM. On the other hand, the period of $510 \mathrm{~s}$ found in the temperature data of GRIPS lies within the uncertainty range of wave (II) observed in the FAIM images $(585 \pm 100 \mathrm{~s})$. This wave structure has a wavelength of about $20 \mathrm{~km}$ and should be resolved by the GRIPS instrument. Other periods may correspond to larger-scale waves which are not visible in the FAIM images itself. However, the disappearing temperature oscillation can tentatively be interpreted as a breakdown of a larger-scale wave into smaller structures, which are clearly visible in the FAIM images and probably decay into KHIs. 
Table 1. Summary of the determined wave parameters identified in Fig. 9. Wave structure (I) is separated into (I.1), lasting until 21:54 UTC and (I.2), emerging at 21:54 UTC. $\lambda$ is the horizontal wavelength once determined in position space $\lambda_{1}$ (see Fig. 9a and b), once in frequency domain $\lambda_{2}$ (Fig. 9c and d). $\Delta t$ is the lifetime determined in the frequency domain (Fig. 9c and d), $T$ is the wave period (determined from Fig. $9 \mathrm{a}$ and $\mathrm{b}$ ) and $v$ the horizontal phase velocity referring to $\lambda_{1}$ and $\lambda_{2}$.

\begin{tabular}{l|cccccc}
\hline & $\lambda_{1}(\mathrm{~km})$ & $\lambda_{2}(\mathrm{~km})$ & $\Delta t(\mathrm{~min})$ & $T(\mathrm{~s})$ & $v_{1}\left(\mathrm{~m} \mathrm{~s}^{-1}\right)$ & $v_{2}\left(\mathrm{~m} \mathrm{~s}^{-1}\right)$ \\
\hline wave (I.1) & $7.0 \pm 2$ & $6.8(6.4-7.2)$ & 18 & $1400 \pm 100$ & $5.0 \pm 1.8$ & $4.9 \pm 0.6$ \\
wave (I.2) & - & $9.0(8.4-9.9)$ & 2 & - & - & - \\
wave (II) & $20.2 \pm 2$ & $24(20-36)$ & 35 & $585 \pm 100$ & $34.5 \pm 9.3$ & $39.3 \pm 21$ \\
\hline
\end{tabular}
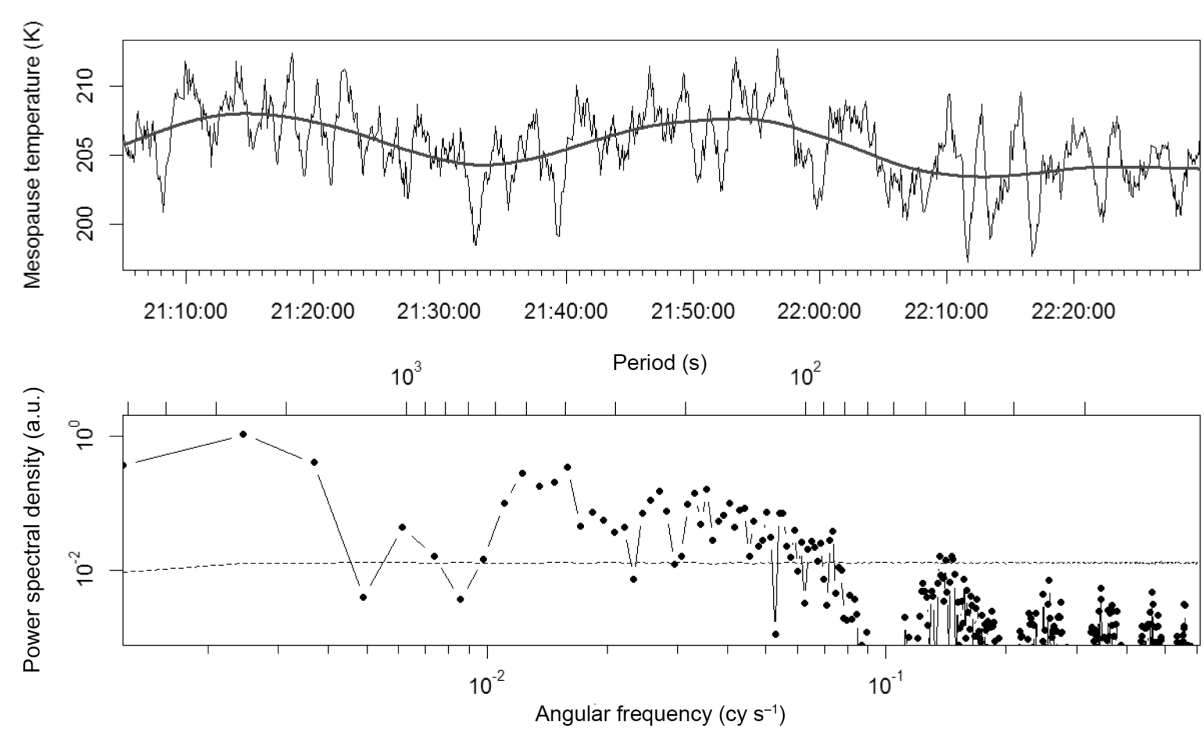

Figure 10. Upper panel: 1 min running averages of the time series of GRIPS 13 measuring in parallel direction with FAIM 1. A smooth spline is drawn as a thick line to guide the eye and show a long-period wave structure which dissolves at the end of the shown interval. Bottom panel: Fourier transform of the temperature time series. Periods of 2500, 1000, 510 and $390 \mathrm{~s}$ are visible in the range of higher periods. The dashed line gives the 0.95 significance. See text for further details.

\section{Summary and conclusions}

We developed the new airglow imager FAIM 1 based on an InGaAs detector, sensitive to the bright $\mathrm{OH}$ emissions between 900 and $1650 \mathrm{~nm}$. Thus, two frames per second can be acquired at a spatial resolution of $200 \mathrm{~m}$ with current optics. Important features of the instrument, in particular a noise level of only 25 counts for a sensor temperature of $235 \mathrm{~K}$, are determined, while signal levels are typically around 850 .

The processing chain, e.g. geographical correction of the images for the standard set-up with $45^{\circ}$ zenith angle are presented. The data of FAIM 1 are compared to GRIPS airglow spectrometric observations Schmidt et al., 2013). In comparison with two such GRIPS instruments, one recording the $1.27 \mu \mathrm{m} \mathrm{O} \mathrm{O}_{2}(0-0)$ emission and one recording the bright $\mathrm{OH}(3-1)$-and $\mathrm{OH}(4-2)$ emissions, it was shown that $\mathrm{O}_{2}$ dominates the FAIM 1 data only during a rather short period of time after sunset. It is worth noting that this time period is shorter than the chemical lifetime of $1 \mathrm{~h}$ of the excited $\mathrm{O}_{2}$ state. During clear sky conditions the broadband FAIM 1 data show a high correlation of up to 0.99 with the spectrally resolved GRIPS data.

A case study was performed in order to demonstrate the capability of the instrument to observe smaller-scale gravity wave structures in the $\mathrm{OH}$ airglow layer at about $87 \mathrm{~km}$ altitude. During the night from 3 to 4 October 2014 two prominent wave structures were identified and analysed. A smaller structure with about $7 \mathrm{~km}$ horizontal wavelength is probably part of a dissipation process of a larger one with about $20 \mathrm{~km}$ horizontal wavelength. The small wave has a nearly perpendicular direction of propagation compared to the larger one and a short lifetime of $20 \mathrm{~min}$. It is therefore tentatively interpreted as a so-called ripple structure.

Where the superposition of both waves takes place, one can see even smaller structures in the order of about $2 \mathrm{~km}$, which we assume to be Kelvin-Helmholtz instability billows (compare Fritts et al. (2014); Hecht et al., 2014).

In the FAIM 1 data of 2014 there are more examples for billow and ripple phenomena which are not yet analysed in such great detail. It is an open question whether these phe- 
nomena are actually common or the instrument's set-up and site offer a unique possibility to study them. Further investigation and statistics from more nights and at other sites may help to answer this question.

Acknowledgements. This work is funded by the Bavarian State Ministry of the Environment and Consumer Protection by grant number TUS01UFS-67093. The investigated data are archived at WDC-RSAT (World Data Center for Remote Sensing of the Atmosphere). The observations are part of NDMC (http://wdc.dlr.de/ndmc).

Edited by: W. Ward

\section{References}

Adams, G. W., Peterson, A. W., Brosnahan, J. W., and Neuschaefer, J. W.: Radar and optical observations of mesospheric wave activity during the lunar eclipse of 6 July 1982, J. Atmos. Terr. Phys., 50, 11-17, 19-20, doi:10.1016/0021-9169(88)90003-7, 1988.

Baker, D. J. and Stair Jr., A. T.: Rocket Measurements of the Altitude Distributions of the Hydroxyl Airglow, Physica Scripta, 37, 611-622, 1988.

Bovensmann, H., Burrows, J. P., Buchwitz, M., Frerick, J., Noel, S., and Rozanov, V. V.: SCIAMACHY: Mission Objectives and Measurement Modes, J. Atmos. Sci., 56, 127-150, doi:10.1175/1520-0469(1999)056<0127:SMOAMM>2.0.CO;2, 1999.

Chu, X., Gardner, C. S., and Franke, S. J.: Nocturnal thermal structure of the mesosphere and lower thermosphere region at Maui, Hawaii $\left(20.7^{\circ} \mathrm{N}\right)$, and Starfire Optical Range, New Mexico $\left(35^{\circ} \mathrm{N}\right)$, J. Geophys. Res., 110, D09S03, doi:10.1029/2004JD004891, 2005.

Dunker, T., Hoppe, U., Feng, W., Plane, J. M., and Marsh, D. R.: Mesospheric temperatures and sodium properties measured with the ALOMAR Na lidar compared with WACCM, J. Atmos. Sol.Terr. Phy., 127, 111-119, doi:10.1016/j.jastp.2015.01.003, 2015.

Fritts, D. C. and Alexander, M. J.: Gravity wave dynamics and effects in the middle atmosphere, Rev. Geophys., 41, 1003, doi:10.1029/2001RG000106, 2003.

Fritts, D. C., Wan, K., Werne, J., Lund, T., and Hecht, J. H.: Modeling the implications of Kelvin-Helmholtz instability dynamics for airglow observations, J. Geophys. Res.-Atmos., 119, 88588871, doi:10.1002/2014JD021737, 2014.

Hecht, J. H.: Instability layer and airglow imaging, Rev. Geophys., 42, RG1001, doi:10.1029/2003RG000131, 2004.

Hecht, J. H., Walterscheid, R. L., Hickey, M. P., Rudy, R. J., and Liu, A. Z.: An observation of a fast external atmospheric acousticgravity wave, J. Geophyis. Res., 107, ACL 12-1-ACL 12-12, doi:10.1029/2001JD001438, 2002.

Hecht, J. H., Wan, K., Gelinas, L. J., Fritts, D., Walterscheid, R., Rudy, R., Liu, A., Franke, S. J., Vargas, F., Pautet, P. D., Taylor, M., and Swenson, G.: The life cycle of instability features measured from the Andes Lidar Observatory over Cerro Pachon on 24 march 2012, J. Geophys. Res.-Atmos., 119, 8872-8898, doi:10.1002/2014JD021726, 2014.
Meinel, A. B.: OH Emission band in the spectrum of the night sky I, Am. Astr. Soc., 111, p. 555, doi:10.1086/145296, 1950.

Moreels, G., Clairemidi, J., Faivre, M., Mougin-Sisini, D., Kouahla, M. N., Meriwether, J. W., Lehmacher, G. A., Vidal, E., and Veliz, O.: Stereoscopic imaging of the hydroxyl emissive layer at low latitudes, Planet. Space Sci., 56, 1467-1479, doi:10.1016/j.pss.2008.04.012, 2008.

Mukherjee, G., Parihar, N., Ghodpage, R., Patil, P., et al.: Studies of the wind filtering effect of gravity waves observed at Allahabad $\left(25.45^{\circ} \mathrm{N}, 81.85^{\circ} \mathrm{E}\right)$ in India, Earth Planets Space, 62, 309-318, doi:10.5047/eps.2009.11.008, 2010.

Mulligan, F. J. and Galligan, J. M.: Mesopause temperatures calculated from the $\mathrm{O}_{2}\left(a^{1} \Delta_{g}\right)$ twilight airglow emission recorded at Maynooth $\left(53.2^{\circ} \mathrm{N}, 6.4^{\circ} \mathrm{W}\right)$, Ann. Geophys., 13, 558-566, doi:10.1007/s00585-995-0558-1, 1995.

Nakamura, T., Higashikawa, A., Tsuda, T., and Matsushita, Y.: Seasonal variations of gravity wave structures in $\mathrm{OH}$ airglow with a CCD imager at Shigaraki, Earth Planets Space, 51, 897-906, doi:10.1186/BF03353248, 1999.

Noll, S., Kausch, W., Kimeswenger, S., Unterguggenberger, S., and Jones, A. M.: $\mathrm{OH}$ populations and temperatures from simultaneous spectroscopic observations of 25 bands, Atmos. Chem. Phys., 15, 3647-3669, doi:10.5194/acp-15-3647-2015, 2015.

Pautet, P.-D., Taylor, M. J., W. R. Pendleton, J., Zhao, Y., Yuan, T., Esplin, R., and McLain, D.: Advanced mesospheric temperature mapper for high-latitude airglow studies, Appl. Optics, 53, 59345943, doi:10.1364/AO.53.005934, 2014.

Pilger, C., Schmidt, C., Streicher, F., Wüst, S., and Bittner, M.: Airglow observations of orographic, volcanic and meteorological infrasound signatures, J. Atmos. Sol.-Terr. Phy., 104, 55-66, doi:10.1016/j.jastp.2013.08.008, 2013.

Roach, F. E. and Gordon, J. L.: The light of the nightsky, Springer Verlag, Berlin-Heidelberg, Germany, doi:10.1086/142513, 1973.

Rousselot, P., Lidman, C., Cuby, J.-G., Moreels, G., and Monnet, G.: Night-sky spectral atlas of $\mathrm{OH}$ emission lines in the nearinfrared, Astron. Astrophys., 354, 1134-1150, 2000.

Sato, K.: A statistical study of the structure, saturation and sources of inertio-gravity waves in the lower stratosphere observed with the MU radar, J. Atmos. Terr. Phys., 56, 755-774, doi:10.1016/0021-9169(94)90131-7, 1994.

Schmidt, C., Höppner, K., and Bittner, M.: A ground-based spectrometer equipped with an InGaAs array for routine observations of $\mathrm{OH}(3-1)$ rotational temperatures in the mesopause region, J. Atmos. Sol.-Terr. Phys., 102, 125-139, doi:10.1016/j.jastp.2013.05.001, 2013.

Shiokawa, K., Katoh, Y., Satoh, M., Ejiri, M., Ogawa, T., Nakamura, T., Tsuda, T., and Wiens, R. H.: Development of Optical Mesosphere Thermosphere Imagers (OMTI), Earth Planets Space, 51, 887-896, doi:10.1186/BF03353247, 1999.

Smith, A. K.: Global Dynamics of the MLT, Surv. Geophys., 33, 1177-1230, doi:10.1007/s10712-012-9196-9, 2012.

Smith, S., Baumgardner, J., and Mendillo, M.: Evidence of mesospheric gravity-waves generated by orographic forcing in the troposphere, Geophys. Res. Lett., 36, L08807, doi:10.1029/2008GL036936, 2009.

Taguchi, M., Ejiri, M., and Tomimatsu, K.: A new all-sky optics for aurora and airglow imaging, National Institute of Polar Research, Tokyo, Japan, Tech. rep., 2004. 
Taylor, M. J. and Hapgood, M. A.: On the origin of ripple-type wave structure in the $\mathrm{OH}$ nightglow emission, Planet. Space Sci., 38, 1421-1430, doi:10.1016/0032-0633(90)90117-9, 1990.

Taylor, M. J., Bishop, M. B., and Taylor, V.: All-sky measurements of short period waves iimage in the $\mathrm{OI}(557.7 \mathrm{~nm}), \mathrm{Na}(589.2 \mathrm{~nm})$ and near infrared $\mathrm{OH}$ and $\mathrm{O}_{2}(0,1)$ nightglow eemission during the ALOHA-93 campaign, Geophys. Res. Lett., 22, 2833-2836, doi:10.1029/95GL02946, 1995.

Taylor, M. J., Pendleton Jr., W. R., Gardner, C. S., and States, R. J.: Comparison of terdiurnal tidal oscillations in mesospheric $\mathrm{OH}$ rotational temperature and $\mathrm{Na}$ lidar temperature measurements at mid-latitudes for fall/spring conditions, Earth Planets Space, 51, 877-885, doi:10.1186/BF03353246, 1999. van Rhijn, P. J.: On the brightness of the sky at night and the total amount of starlight, Publications of the Astronomical Laboratory at Groningen, Groningen, the Netherlands, 1921.

von Savigny, C.: Variability of OH(3-1) emission altitude from 2003 to 2011: Long-term stability and universality of the emission rate-altitude relationship, J. Atmos. Sol.-Terr. Phy., 127, 120 128, doi:10.1016/j.jastp.2015.02.001, 2015.

Yamada, Y., Fukunishi, H., Nakamura, T., and Tsuda, T.: Breaking of small-scale gravity wave and transition to turbulence observed in $\mathrm{OH}$ airglow, Geophys. Res. Lett., 28, 2153-2156, doi:10.1029/2000GL011945, 2001. 